\title{
Relocalización de paradas de transporte público para ser incorporado dentro planes de contingencia como resultado de la construcción de obras de mejora vial
}

\author{
Marcos Medina-Tapia \\ Académico, Universidad de Santiago de Chile, Chile \\ Cristian Baeza \\ Consultor, Universidad de Santiago de Chile, Chile \\ Daniel Arancibia \\ Académico, Universidad de Santiago de Chile, Chile \\ Francesc Robusté \\ Catedrático de Transporte, Universidad Politécnica de Cataluña, España
}

\section{RESUMEN}

Las paradas de transporte público (TP), tanto en su distribución como en su localización, son generalmente analizadas desde un punto de vista macroscópico, utilizando modelos analíticos para observar tanto una línea de buses, en particular, o la red de transporte público, en general. Sin embargo, la importancia y complejidad de lograr una buena operación de las paradas de transporte público y en particular lograr que éstas tengan una buena accesibilidad, requiere de un análisis más detallado.

Actualmente no existen desarrollos metodológicos que solucionen este problema, menos aún en el caso de proyectos viales de mejora de la infraestructura urbana que implican una intervención importante de un sector de la ciudad, por lo que en este trabajo se propone una metodología de localización de paradas de transporte público que a través de un modelo matemático de optimización permita ubicar dichos paraderos provisorios a lo largo de las calles propuestas en un Plan de Desvíos de manera tal de optimizar la accesibilidad de los usuarios al transporte público.

Esta metodología fue aplicada a un caso real de estudio en la intersección de las avenidas 5 de Abril - Esquina Blanca con Avenida Del Ferrocarril de Santiago, Chile, y los resultados muestran que se puede mantener y, en algunos casos, mejorar el nivel de servicio del transporte público desde el punto de vista de la accesibilidad al mismo. Por lo tanto, se demuestra la importancia y el aporte que esta metodología puede tener para mejorar la planificación de los Planes de Desvío por obras de mejoramiento vial al incorporar la variable accesibilidad al transporte público. 


\section{INTRODUCCIÓN Y OBJETIVO}

\subsection{Presentación del problema}

La congestión vehicular ha sido considerada como un problema complejo y dinámico. Es por esto que el estudio del flujo vehicular tiene como objetivo principal, ayudar a comprender el problema de la congestión de tránsito, servir como herramienta de apoyo para la toma de decisiones y plantear soluciones para dicho problema (May, 1990). En este sentido, los primeros intentos de desarrollar un modelo matemático para describir el flujo vehicular datan de los años 30, pero a pesar de eso, hasta el día de hoy aún no existe un modelo satisfactorio o una teoría matemática general para describir las condiciones reales del tráfico (Papageorgiou et al., 2009). Esto se debe a que la interacción vehicular no responde solamente a la aplicación de las leyes de la física, sino que también responde a la influencia del actuar psicológico de los conductores, es decir, al comportamiento humano (Ioannou y Bose, 2000).

En este escenario, las herramientas computacionales de modelación de tránsito constituyen una alternativa muy efectiva para poder realizar evaluaciones de distintas situaciones en las condiciones del tráfico de una ciudad, comuna o incluso de una vía en particular. Dentro de estas herramientas se encuentra la Microsimulación, que permite evaluar las acciones individuales de cada vehículo a intervalos de tiempo determinados, incorporando atributos generales del comportamiento de los usuarios.

Los paraderos de transporte público, su distribución y localización, son principalmente analizados en la literatura desde un punto de vista macroscópico y se usan modelos analíticos para observar tanto una línea de buses (recorrido), o la red de transporte público completa. Sin embargo, la importancia y complejidad de lograr una buena operación de los paraderos de transporte público requiere de una planificación más detallada, por lo tanto, se hace necesario plantear un modelo que se ocupe exclusivamente de este problema, para así poder comprender y planificar de mejor manera el comportamiento del tráfico en general, y particularmente del transporte público.

Esto es debido a que diversos autores consideran que los paraderos de buses son uno de los principales problemas que explican la congestión vehicular, ya que no sólo representan el lugar desde donde los usuarios de transporte público acceden al sistema de tránsito sino que son también un factor determinante en la velocidad promedio que alcanzan los buses. A pesar de esto, en el pasado, los esfuerzos por desarrollar políticas de transporte estuvieron orientados, principalmente, a modelar el movimiento de los automóviles, y la representación y desarrollo de herramientas para simular el comportamiento del transporte público fue mínimo.

Actualmente, en Chile se utiliza el Manual de Diseño y Evaluación Social de Proyectos de Vialidad Urbana (MESPIVU), el cual fue desarrollado por el Ministerio de Transporte y 
Telecomunicaciones de Chile en 1988, el cual constituye la principal metodología, a nivel chileno, para el análisis y evaluación de proyectos de vialidad urbana. Sin embargo, este manual no profundiza en la relocalización de los paraderos de transporte público que se verán afectados por los proyectos de vialidad.

\subsection{Objetivo}

El objetivo de la presente investigación es desarrollar una metodología que permita la localización óptima de paraderos provisorios de transporte público en vías urbanas alternativas que han sido propuestas a raíz de un plan de contingencia por la construcción de obras de mejoramiento de infraestructura vial, y que permita ser aplicable por los organismos y autoridades pertinentes, para cualquier proyecto de este tipo, que si bien es temporal, puede tener una construcción que se prolongue al menos por un par de años.

Para el cumplimiento del objetivo principal, se requiere construir un modelo de optimización matemática para la localización de los paraderos de transporte público, junto con la implementación de la microsimulación para observar el comportamiento vial y peatonal, de los paraderos relocalizados dentro del sistema de transporte urbano, permitiendo el análisis de los escenarios propuestos y el análisis de sensibilidad de la metodología frente a las variables utilizadas. Para complementar la información requerida por el modelo, se realizaron mediciones de flujo vehicular y se realizaron encuestas para conocer el comportamiento de los usuarios en paraderos en el área de aplicación.

\subsection{Investigación previa}

La densidad y localización de paraderos de transporte público influyen de manera directa en la accesibilidad que tienen los pasajeros de los buses al sistema de transporte urbano, e influyen de manera significativa en el nivel de tránsito y de servicio que pueden ofrecer los recorridos de transporte público (Medina, 2011).

De acuerdo a Chien et al. (2004), los estudios de optimización de paraderos se pueden clasificar en tres categorías distintas. La primera categoría agrupa a los modelos que usan distribuciones de demanda simplificada y que están enfocados en la localización de paraderos utilizando distribuciones de demanda simple, considerando que la demanda no varía en el tiempo. La segunda categoría incluye los modelos que optimizan la operación y la distancia entre paraderos y que se enfocan en la optimización conjunta del diseño del servicio y el espaciamiento entre paraderos de forma simultánea (Medina et al., 2013). En la tercera categoría, se encuentran los modelos que consideran que la demanda varía en el tiempo y que optimizan el sistema de transporte público en función de esto.

El enfoque de este trabajo considera la tercera categoría, donde la demanda de pasajeros varía en el tiempo, el cual se desarrolla a través de la simulación. La simulación del tráfico busca representar el comportamiento del flujo vehicular mediante la construcción de un 
modelo computacional y cómo este varía en el tiempo. En lo que concierne a la investigación de modelos de transporte, estos pueden clasificarse en tres tipos (Dowling et al., 2004):

a) Modelos de Simulación Macroscópica: Describen el flujo vehicular a través de una red de transporte mediante el uso de variables como la densidad de vehículos, el flujo y la velocidad promedio.

b) Modelos de Simulación Mesoscópico: La unidad de flujos utilizada es el vehículo individual, pero para los movimientos se considera el enfoque macroscópico. Estos modelos combinan las propiedades de los modelos macroscópicos y microscópicos.

c) Modelos de Simulación Microscópica: Estos modelos simulan el movimiento individual de los vehículos e incorporan sub-modelos para representar la aceleración, las variaciones de velocidad, cambios de pistas, espacios disponibles, entre otros, con el fin de describir el comportamiento de un vehículo con respecto a los demás y a la infraestructura existente. Este es el enfoque que tiene el presente trabajo.

En los últimos años se han realizado numerosas investigaciones con respecto al tema de la microsimulación, centradas principalmente en los softwares existentes en el mercado y en los modelos utilizados para representar situaciones de tráfico en diversas situaciones (Gipps, 1981; Sykes, 2007; Hidas et al., 2009; Papageorgiou, 2009; Cortés, 2010). Los softwares más utilizados son Paramics, Aimsun y Vissim (Olstam y Tapani, 2004; Ronaldo, 2012). En el caso de esta investigación, el software utilizado corresponde a Aimsun. Aimsun es un software de modelación de transporte desarrollado por Transport Simulation System (TSS) que es capaz de reproducir las condiciones de tráfico reales de cualquier red vial en un computador. Además, integra el módulo Legion el cual permite microsimular el comportamiento de los peatones dentro de la red de transporte.

No son muchos los trabajos que abordan centralmente la localización de paradas con un enfoque de microsimulación. Uno de éstos es el desarrollo de Moura et al. (2012), quienes realizan una investigación que analiza la localización óptima de paraderos de transporte público a través de un modelo de dos etapas. En la primera etapa, los paraderos son localizados estratégicamente a nivel macroscópico a lo largo de todo el sistema de transporte público minimizando el costo social de dicha localización. Dichos costos están asociados a los costos de los usuarios (tiempo de acceso, de espera y tiempo de viaje) y las costos en los que incurren los operadores del transporte público (costos directos e indirectos). La segunda etapa del modelo consiste en afinar la localización de los paraderos, que fueron ubicados macroscópicamente, a través de un modelo de microsimulación que maximice el servicio otorgado por los operadores de transporte público. Los resultados obtenidos por los investigadores en este estudio demuestran la importancia de la microsimulación como herramienta para poder mejorar los niveles de servicios del transporte público en general, basados en una óptima localización de los paraderos. 


\section{MARCO METODOLÓGICO}

\subsection{Metodología propuesta}

Las obras de mejoramiento de una avenida, calle principal o secundaria, siempre implican una alteración y/o interrupción del flujo vehicular normal de dicha vía. Debido a esto, los encargados de la ejecución de dichas obras (gobierno o autoridades locales, en conjunto con los ministerios relacionados) optan por proponer vías alternativas para no alterar en demasía el normal tránsito de los vehículos. La implementación de estas vías tiene un costo asociado, tanto para los encargados de las obras que deben instalar infraestructura provisoria para las nuevas paradas de transporte público, etc., como para los usuarios de estas vías, como los peatones, que ven trasladados sus habituales puntos de acceso al transporte público, y que deben desplazarse a la nueva ubicación, que en la mayoría de los casos, implica un costo en el tiempo para trasladarse a la nueva localización de los paraderos.

A continuación se propone una metodología que pretende optimizar dicho procedimiento a través de un modelo que relocalice los nuevos paraderos de manera tal que minimice los costos de instalación y los costos de traslado de los usuarios de dichos paraderos, asociados a los tiempos de desplazamiento. La Figura 1 presenta el desarrollo metodológico a través de cuatro etapas: construcción del modelo, modelación, análisis y propuesta de localización. En la etapa de modelación se hace interactuar un modelo de programación matemática que se presenta en el siguiente punto, con un proceso de microsimulación para evaluar el comportamiento de los paraderos en sus nuevas localizaciones. Si no es satisfactorio, se actualizan los parámetros y se modela nuevamente.

\subsection{Modelo de localización}

A continuación se presenta un modelo de localización de paraderos de transporte público, que asume una determinada red de transporte y la demanda existente de dichos servicios. La idea principal de este modelo es que permita localizar paraderos dentro de un conjunto de potenciales ubicaciones, minimizando el costo de instalación de dichos paraderos desde el punto de vista de los usuarios y de los operadores del sistema de transporte público. Para el planteamiento de este modelo se tendrá en cuenta una red dada de transporte, $G=(N, A)$, donde el conjunto A corresponde a los arcos dados por las calles de la ciudad que sirven para conectar los paraderos y el conjunto $\mathrm{N}$ de los nodos de dicha red correspondiente al grupo de paraderos existentes y los potenciales paraderos ubicados en las vías alternativas a los trabajos de remodelación. Además existe un conjunto L de buses que son utilizados para satisfacer la demanda de transporte de los pasajeros de dichos paraderos. 


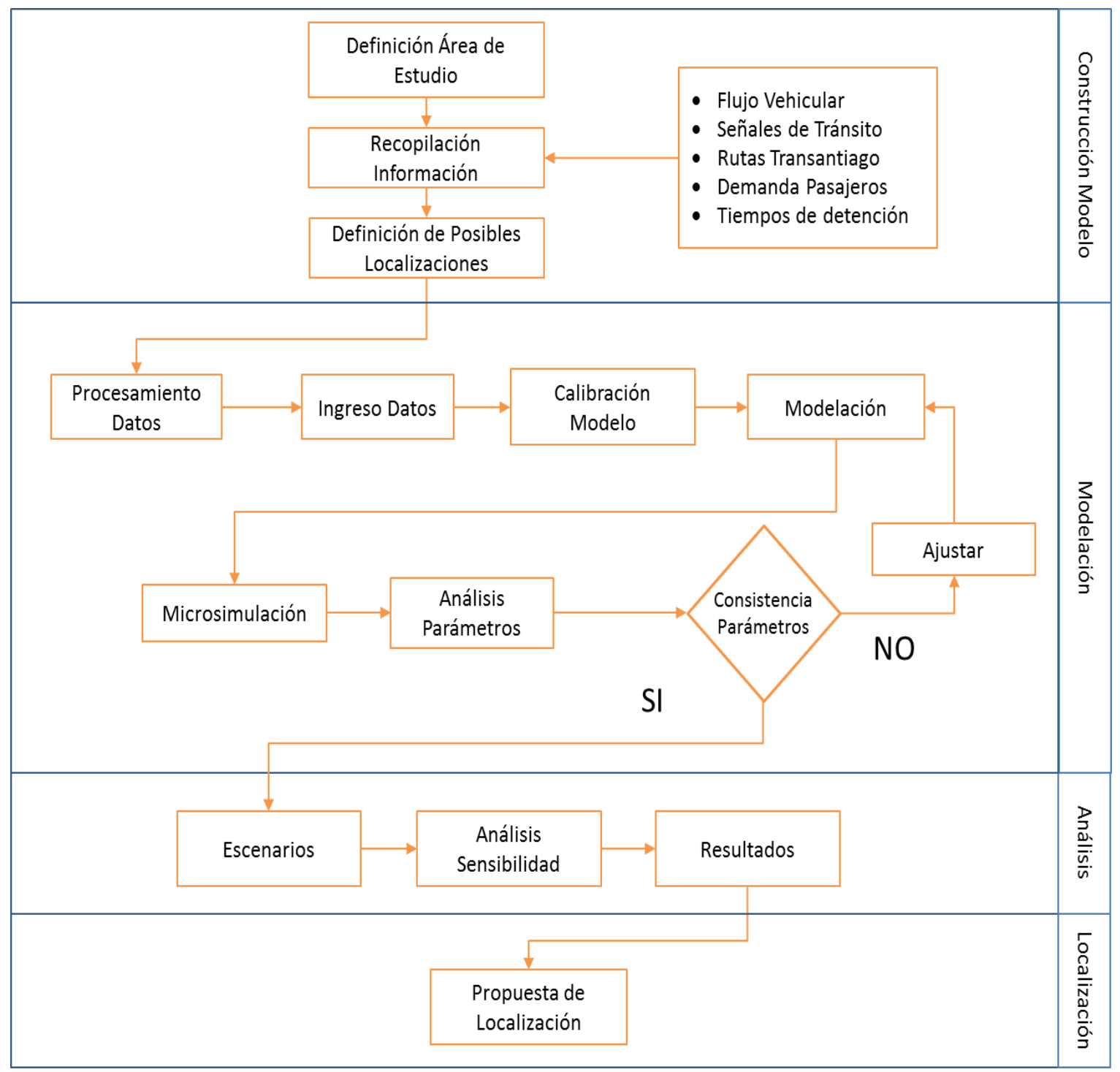

Fig. 1 - Metodología de localización de paradas de transporte público

A continuación se presentan las variables de decisión del modelo que determinan la localización del paradero y la cantidad de pasajeros asignados a dichos paraderos.

$y_{j}^{k}$ : Variable entera binaria que toma el valor 1 si el paradero de tamaño $k$ se localiza en $\mathrm{j}$ $\mathrm{y}$ toma valor 0 si no se localiza, para todo $\mathrm{j} \epsilon \mathrm{N}$.

$\mathrm{x}_{\mathrm{ij}}^{\operatorname{lm}}$ : Variable entera de asignación de pasajeros que vienen desde la zona i y que tiene que dirigirse al nuevo paradero $\mathrm{j}$ para subir al recorrido 1 en el período $m$, para todo $i, j \in N$.

$\mathrm{z}_{\mathrm{ji}}^{\mathrm{lm}}$ : Variable entera de asignación de pasajeros que van hacia la zona i provenientes del nuevo paradero $\mathrm{j}$ al bajar del recorrido $\mathrm{l}$ en el período $\mathrm{m}$, para todo $\mathrm{i}, \mathrm{j} \in \mathrm{N}$.

Además es necesario definir una serie de parámetros necesarios para el funcionamiento del modelo, los cuales se presentan a continuación. 
$\mathrm{ts}_{\mathrm{ij}}$ : Este parámetro mide el tiempo de desplazamiento de los pasajeros desde zona i de origen del viaje del pasajero hasta el paradero $\mathrm{j}$ [horas].

$\mathrm{tb}_{\mathrm{ij}}$ : Este parámetro mide el tiempo de desplazamiento de los pasajeros desde el paradero $\mathrm{j}$ en el que descienden del transporte público hasta la zona i de destino [horas].

$\theta_{\mathrm{c}}^{\mathrm{k}}$ : Costo de construcción del nuevo paradero de tamaño $\mathrm{k}$ [\$].

$\theta_{\mathrm{a}}$ : Costo social del tiempo de caminata de los pasajeros al trasladarse a los nuevos paraderos [\$/pasajero*hora].

$\mathrm{O}_{\mathrm{i}}^{\operatorname{lm}}$ : Número de pasajeros que suben al recorrido 1 en el período m provenientes de la zona i [pasajeros/hora].

$\mathrm{D}_{\mathrm{i}}^{\operatorname{lm}}$ : Número de pasajeros que bajan del recorrido 1 en el período $\mathrm{m}$ y que se dirigen a la zona i [pasajeros/hora].

$\mathrm{q}_{\mathrm{j}}^{\mathrm{k}}$ : Capacidad de pasajeros que tiene el paradero nuevo $\mathrm{j}$ para el tamaño $\mathrm{k}$ [pasajeros/hora].

$\mathrm{Q}_{\mathrm{j}}^{\mathrm{k}}$ : Capacidad del paradero nuevo $\mathrm{j}$ de tamaño $\mathrm{k}$ de recibir buses en cola sin alterar el normal funcionamiento de éste [buses/hora].

$\mathrm{w}_{\mathrm{j}}^{\operatorname{lm}}$ : Frecuencia de los buses del recorrido 1 que llegan al paradero nuevo $\mathrm{j}$ en el período $\mathrm{m}$ [buses/hora].

M:Es un parámetro muy grande, que puede tomar cualquier valor mayor que cero.

Así, el modelo busca minimizar los costos totales de localización asociados a los usuarios, en cuanto al tiempo que demoran en trasladarse desde su lugar de origen hacia el nuevo paradero propuesto, o viceversa. Además se consideran los costos de construcción asociados al nuevo paradero. Además, restringe las localizaciones a la capacidad de éstos de recibir pasajeros de acuerdo a su tamaño, y a la demanda de pasajeros existente en el área de estudio. Así el modelo propuesto se presenta a continuación.

$$
\operatorname{Min} \sum_{\mathrm{j}} \sum_{\mathrm{k}} \mathrm{y}_{\mathrm{j}}^{\mathrm{k}} \theta_{\mathrm{c}}^{\mathrm{k}}+\sum_{\mathrm{i}} \sum_{\mathrm{j}} \sum_{\mathrm{l}} \sum_{\mathrm{m}}\left(\mathrm{ts}_{\mathrm{ij}} \mathrm{x}_{\mathrm{ij}}^{\mathrm{lm}}\right) * \theta_{\mathrm{a}}+\left(\mathrm{tb}_{\mathrm{ji}} \mathrm{z}_{\mathrm{ji}}^{\mathrm{lm}}\right) * \theta_{\mathrm{a}}
$$

s.a.

$$
\begin{gathered}
\sum_{\mathrm{k}} \mathrm{y}_{\mathrm{j}}^{\mathrm{k}}<=1 \quad \forall \mathrm{j} \\
\sum_{\mathrm{j}} \mathrm{x}_{\mathrm{ij}}^{\operatorname{lm}}=O_{i}^{l m} \quad \forall \mathrm{i}, \mathrm{l}, \mathrm{m} \\
\sum_{\mathrm{j}} \mathrm{z}_{\mathrm{ji}}^{\operatorname{lm}}=D_{i}^{l m} \quad \forall \mathrm{i}, \mathrm{l}, \mathrm{m} \\
\sum_{\mathrm{i}} \sum_{\mathrm{l}} \mathrm{x}_{\mathrm{ij}}^{\operatorname{lm}} \leq \sum_{\mathrm{k}} \mathrm{q}_{\mathrm{j}}^{\mathrm{k}} * \mathrm{y}_{\mathrm{j}}^{\mathrm{k}} \quad \forall \mathrm{j}, \mathrm{m} \\
\sum_{\mathrm{i}} \sum_{\mathrm{l}} \mathrm{z}_{\mathrm{ji}}^{\mathrm{lm}} \leq \sum_{\mathrm{k}} \mathrm{M} * \mathrm{y}_{\mathrm{j}}^{\mathrm{k}} \quad \forall \mathrm{j}, \mathrm{m} \\
\sum_{\mathrm{i}} \sum_{\mathrm{l}} \mathrm{w}_{\mathrm{j}}^{\operatorname{lm}} \leq \sum_{\mathrm{k}} \mathrm{Q}_{\mathrm{j}}^{\mathrm{k}} * \mathrm{y}_{\mathrm{j}}^{\mathrm{k}} \quad \forall \mathrm{j}, \mathrm{m}
\end{gathered}
$$

La ecuación (1) busca minimizar los costos de usuario asociados al tiempo de caminata desde el origen hacia el paradero, o viceversa, y los costos de construcción de los nuevos paraderos. La primera restricción (2) establece que si el paradero propuesto es localizado, el modelo 
escoja solo uno de los tamaños k disponibles para elegir. Las siguientes restricciones indican que la demanda de pasajeros que existían en los paraderos, y que van a ser relocalizados, sea reasignada en su totalidad en los paraderos nuevos localizados (3), así como los que bajan de los nuevos paraderos $\mathrm{j}$ desde el recorrido 1 y que se dirigen a la zona i, sea igual al total de pasajeros que antes bajaban en los antiguos paraderos (4). La restricción (5) indica que el número de pasajeros asignados al nuevo paradero no debe superar la capacidad de dicho paradero considerando su tamaño k. La restricción (6) garantiza que los pasajeros solo se bajen en los paraderos localizados de acuerdo a la variable decisión. La última restricción (7) establece que la frecuencia de buses que se detienen en el paradero seleccionado no supere la capacidad del mismo, en cuanto al número de buses que este puede recibir sin que se formen colas en dicho paradero.

Los datos que requiere este modelo provienen de las encuestas y de los conteos de pasajeros. Durante esta etapa se procesó toda esta información digitalizando las encuestas en caso de ser necesario, y tabulando todos los datos de acuerdo a los requerimientos del modelo. Con las localizaciones de los paraderos más los datos de flujo vehicular y peatonal, es posible realizar la microsimulación del área de estudio y así poder observar el comportamiento que tendrán los usuarios del sistema (peatones, vehículos particulares y transporte público) y si efectivamente las localizaciones propuestas ayudan a simplificar el flujo vehicular de la zona o, por el contrario, no ayuda a disminuir la congestión producida por las obras de mejoramiento.

Una vez realizada la microsimulación es posible efectuar un primer análisis de los resultados obtenidos. Este análisis previo consiste en comparar los datos obtenidos por la microsimulación con la información ingresada en el modelo de optimización para verificar que dichos resultados se ajusten a los datos reales ingresados.

El principal parámetro observado en este análisis son los tiempos de caminata de los peatones. Si los tiempos obtenidos de la microsimulación se ajustan a los datos calculados previamente para ingresarlos al modelo, entonces se continúa con la siguiente etapa de la metodología. En caso que los resultados de la microsimulación sean distintos a los datos reales calculados previamente (por ejemplo, que los tiempos de caminata obtenidos de la microsimulación sean mayores a los reales), entonces es necesario realizar un ajuste de los datos, para luego volver a modelar y microsimular, para comparar nuevamente los resultados y determinar si esta vez sí se ajustan a la realidad para así pasar a la siguiente etapa o, por el contrario, es necesario efectuar un nuevo ajuste.

Una vez que los resultados de la simulación se ajustan a la realidad del área de estudio, entonces es posible plantear diversos escenarios de acuerdo a los horarios simulados (punta mañana, punta tarde, fuera de punta). 


\section{APLICACIÓN A CASO DE ESTUDIO}

\subsection{Descripción de área de aplicación}

El caso de estudio de este trabajo se enfoca en las obras de mejoramiento vial ubicadas en la intersección del eje vial Avenida 5 de Abril y Esquina Blanca con Avenida Del Ferrocarril, en el límite entre las comunas de Maipú y Cerrillos, en el sector sur poniente de la Región Metropolitana de Santiago, Chile. Poseen una población conjunta de 540.296 habitantes (Instituto Nacional de Estadísticas, Chile) y cuentan con una superficie total de $156,5 \mathrm{~km}^{2}$.

Además, el 16 de Mayo de 2013 fue anunciado oficialmente, por el Presidente de la República, la habilitación del Tren de Cercanía Santiago Melipilla (Melitren), servicio que unirá Estación Central con Melipilla. El proyecto cuenta con un largo de $61 \mathrm{~km}$ y 11 estaciones que pasan por las comunas de Estación Central, Maipú, Cerrillos, Talagante y Melipilla, entre otras, y se estima que reducirá los tiempos de viaje a 46 minutos. Se espera que este proyecto esté disponible para el año 2016.

El alto crecimiento poblacional que existe en este sector de la Región Metropolitana, especialmente el que ha experimentado la comuna de Maipú en las últimas décadas, ha traído consigo también un aumento en el parque automotriz de dichas comunas (que se suma al crecimiento natural del mismo, proyectado por las autoridades para el país en los próximos años) y la evidente congestión que esto implica, tanto para las arterias principales de estas comunas como las vías secundarias.

Dentro de este contexto, la metodología señalada anteriormente para las vías alternativas planteadas ante las obras de mejoramientos de las Avenidas 5 de Abril y Esquina Blanca, en la intersección con Avenida Del Ferrocarril, aparece como de vital importancia para contribuir a mejorar la congestión vehicular inherente a este tipo de obras y así evitar un caos vehicular mayor al existente en este sector (Figura 2).

La principal vía de este sector es la Avenida 5 de Abril, una de las avenidas más importantes de Maipú y del sector sur de Santiago, Chile. Tiene una extensión de 3,3 kilómetros, desde Avenida El Carmen junto al Templo Votivo de Maipú hasta Avenida San José justo en la intersección con Camino a Melipilla, y atraviesa de Poniente a Oriente las comunas de Maipú y Cerrillos. En 2010 finalizó la construcción de un corredor exclusivo de transporte público a lo largo de la avenida como parte de las obras de Transantiago.

La Avenida Esquina Blanca se encuentra paralela a la Avenida 5 de Abril y va desde la Plaza de Maipú hasta la intersección con el Camino a Melipilla. Esta avenida es utilizada, principalmente, para el tránsito del transporte privado que circula de Poniente a Oriente.

El uso de suelos de la intersección corresponde principalmente a usos de tipo residencial y existen además, algunos colegios en la zona. Además, la intersección se encuentra cercana 
a centros educacionales y al centro cívico de la comuna de Maipú que es polo comercial y de servicios de la zona.

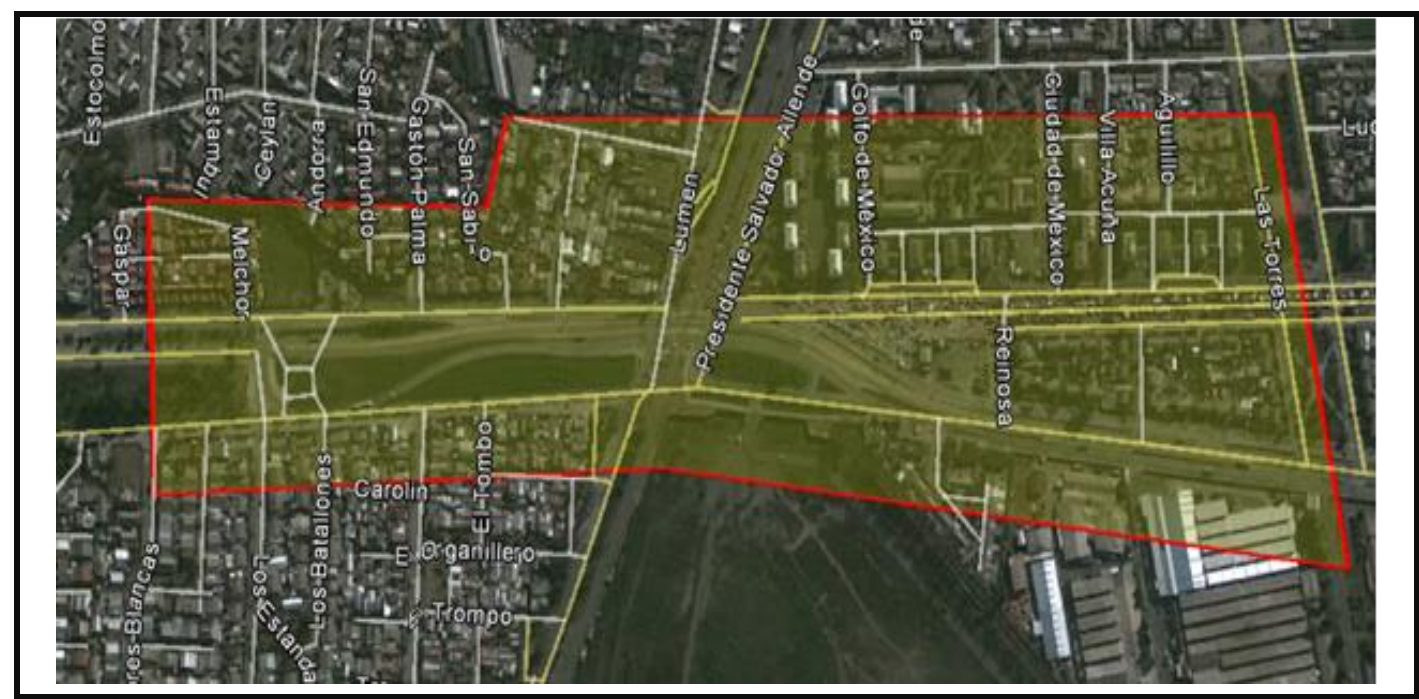

Fig. 2 - Área de Estudio

\subsection{Resultados}

Respecto a la modelación se analizó la situación base, que se modeló con los datos actuales, antes de iniciar las obras y desvíos. Esta será considerada la situación base y servirá para comparar los resultados obtenidos una vez que se hayan hecho los cambios propuestos por el modelo en cuanto a la localización de los paraderos.

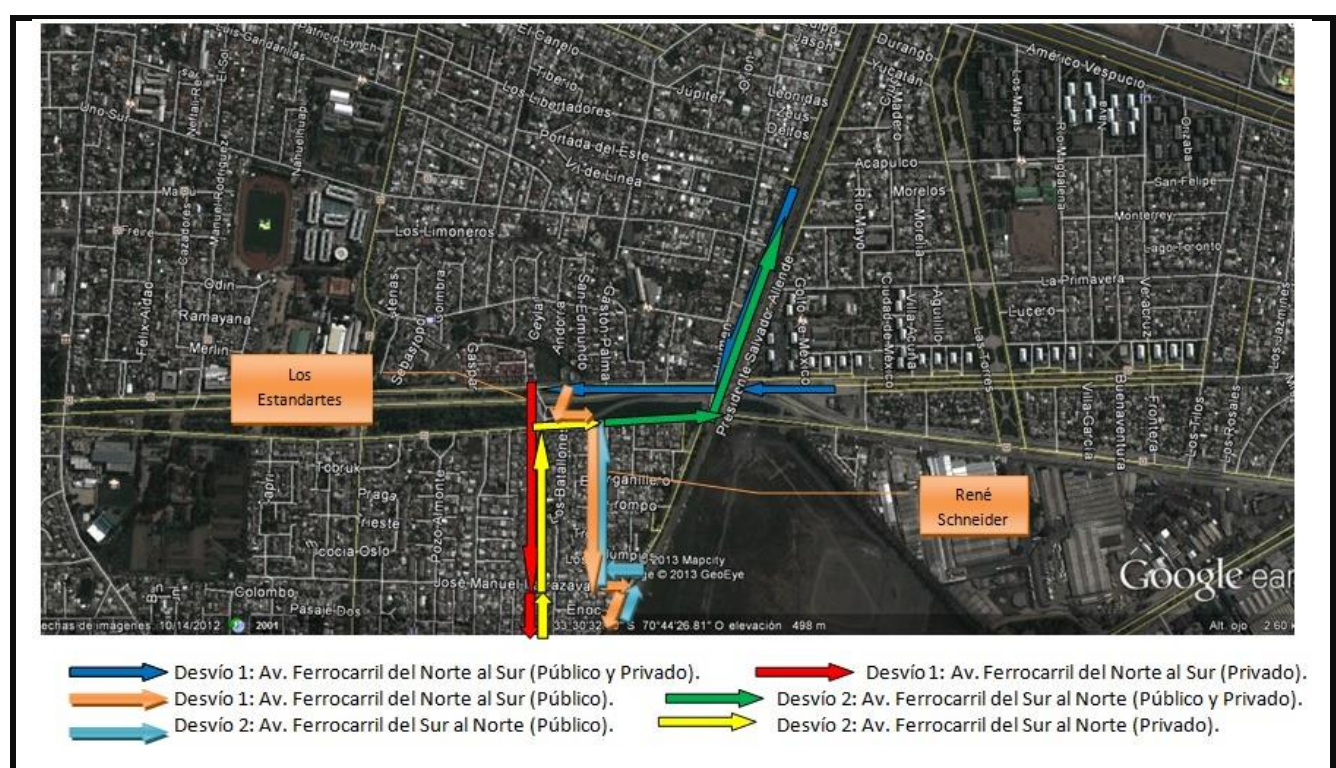

Fig. 3 - Plan de desvíos de la Fase 1

Fuente: Diseño de Proyecto de Ingeniería: Habilitación nudo a desnivel FF. CC. -5 de Abril. Creaxxion Ingenieros Consultores.

En la Figura 3 se presentan los desvíos propuestos para el tráfico de vehículos por Avenida Salvador Allende y Del Ferrocarril, tanto para el transporte público como privado. El 
transporte público que circula por Avenida Del Ferrocarril al sur, será desviado por Avenida 5 de Abril como se observa en la Figura 3. Este desvío afecta particularmente a tres recorridos que circulan por Avenida Esquina Blanca hacia el oriente, cuyos paraderos deberán ser relocalizado para esta primera etapa.

Se realizó una modelación para la Fase 1, introduciendo los desvíos que deben hacer los vehículos que circulan por Avenida Del Ferrocarril, como los nuevos paraderos que deberán utilizar los peatones. Los resultados de esta simulación muestran un aumento en la congestión de la calle Los Estandartes en dirección hacia el sur, donde son desviados los vehículos privados que se dirigen por Avenida Del Ferrocarril. Mientras, el resto de las secciones mantuvieron un flujo vehicular normal, sin congestión. Durante esta fase, y por la naturaleza de los desvíos, la localización del nuevo paradero no afecta el tráfico del área, ya que los recorridos de transporte público son desviados por calles distintas al transporte privado, no afectando el tránsito dentro de la zona intervenida. Particularmente, la cola promedio para los automóviles aumenta considerablemente desde 31.8 vehículos a 56.2 vehículos (Figura 3). La Tabla 1 muestra los cambios producidos por la puesta en marcha del proyecto, manteniéndose los parámetros iniciales.

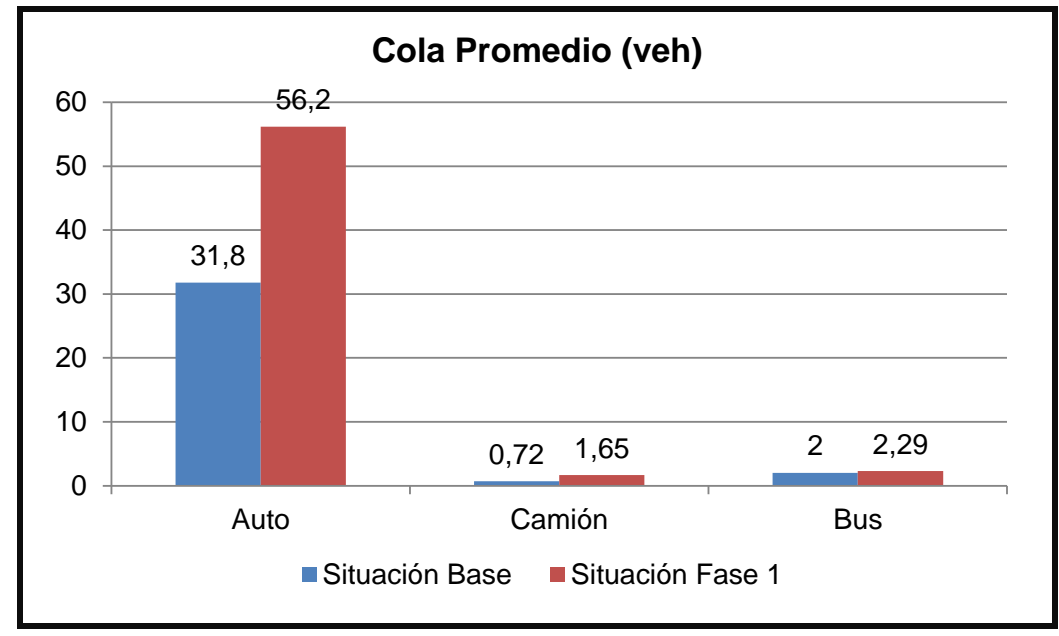

Fig. 4 - Cola Promedio Situación Fase 1 por categoría de vehículos

\begin{tabular}{|l|c|c|}
\hline & Situación Base & Fase 1 \\
\hline Distancia Total Recorrida $(\mathbf{k m})$ & 73.69 & 81.93 \\
\hline Tiempo de Viaje $(\mathbf{s e g})$ & 302.8 & 285.88 \\
\hline Velocidad Promedio $(\mathbf{k m} / \mathbf{h})$ & 5.12 & 5.03 \\
\hline
\end{tabular}

Tabla 1 - Indicadores para Peatones Situación Fase 1

En segundo lugar, se realizó la modelación y microsimulación de los resultados obtenidos para la Fase 2 (Figura 5) del Plan de Desvíos que muestran la congestión de la calle Los Estandartes hacia el sur producto de los desvíos. En este sector se produce un aumento en la congestión producto de los desvíos, el funcionamiento del paradero que se encuentra ubicado ahí no contribuye mayormente a dicho aumento. Si lo analizamos por categoría de vehículos, 
se puede observar que la cola promedio disminuyó para los automóviles de 31.8 a 26.52 vehículos. Sin embargo, tanto para buses como camiones hay un leve aumento, que de todos modos no alcanza a ser significativo. En el caso de los buses, la cola promedio aumentó de 2 a 2.74 vehículos (Figura 6). La Tabla 2 muestra los cambios producidos por la puesta en marcha de la Fase 2 del proyecto, observándose valores similares a la situación base.

En tercer término, se analizó los efectos de una feria libre que se instala los días jueves y domingo, que impide la circulación de vehículos por Avenida Las Torres. Para esto existe otra propuesta de desvíos de la segunda fase de construcción, la cual también fue modelada. En este caso los vehículos tanto privados como de transporte público deben ser desviados por Avenida Los Tilos, seguir por calle La Primavera y luego continuar el recorrido que se definió anteriormente para la Fase 2.

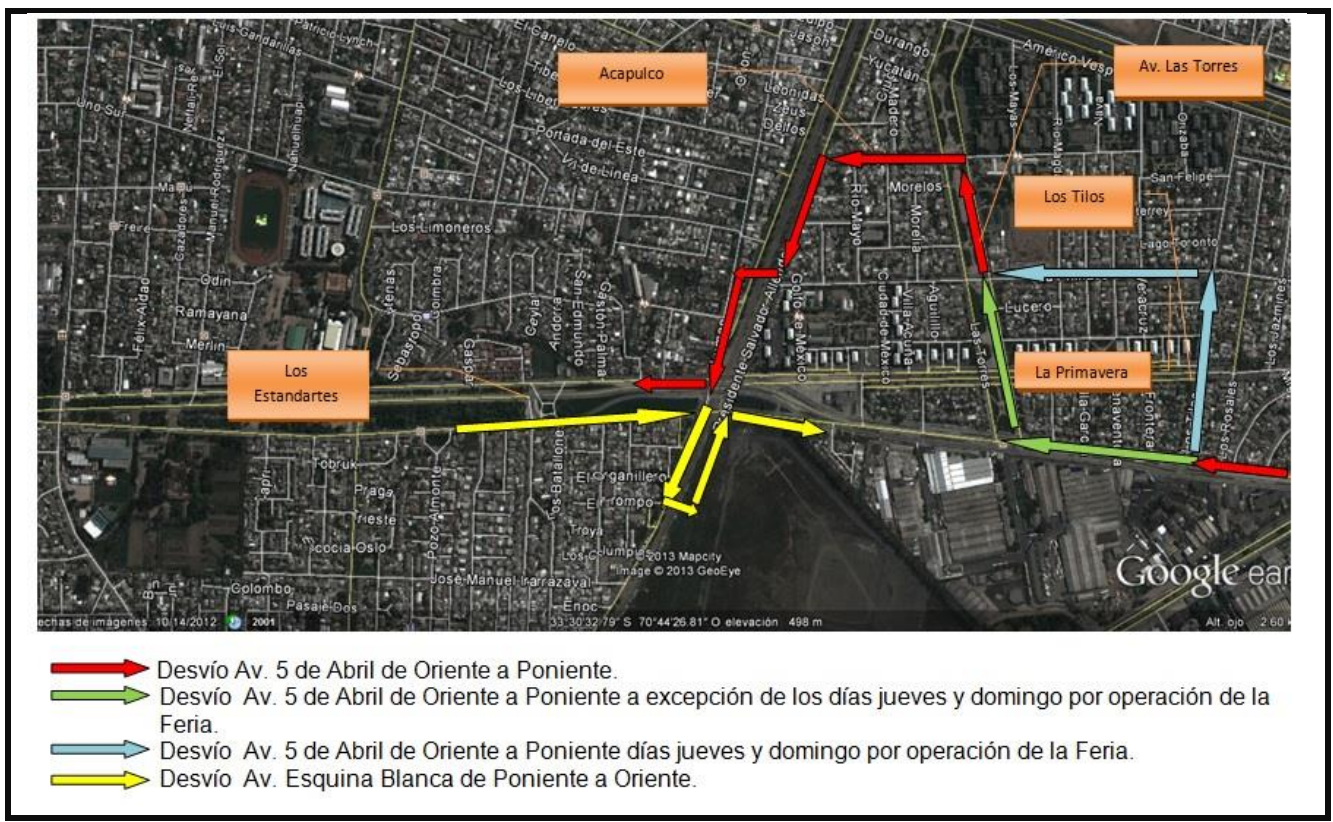

Fig. 5 - Plan de desvíos de la Fase 2

Fuente: Diseño de Proyecto de Ingeniería: Habilitación nudo a desnivel FF. CC. -5 de Abril. Creaxxion Ingenieros Consultores.

\begin{tabular}{|l|c|c|}
\hline & Situación Base & Fase 2 \\
\hline Distancia Total Recorrida $(\mathbf{k m})$ & 73.69 & 131.31 \\
\hline Tiempo de Viaje $(\mathbf{s e g})$ & 302.8 & 298.06 \\
\hline Velocidad Promedio $(\mathbf{k m} / \mathbf{h})$ & 5.12 & 5.23 \\
\hline
\end{tabular}

Tabla 2 - Indicadores para Peatones Situación Fase 2 


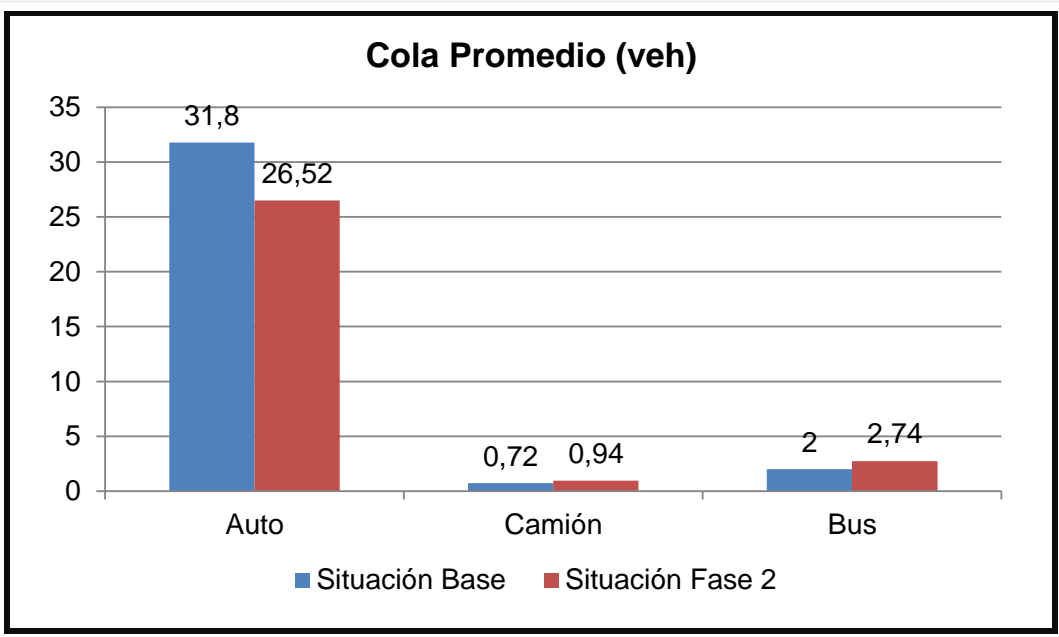

Fig. 6 - Cola Promedio Situación Fase 2 por categoría de vehículos

\subsection{Análisis de sensibilidad}

Para analizar cómo reacciona el modelo de localización de paradas de transporte público frente a distintas circunstancias con las que se puede encontrar al momento de aplicarlo en casos reales, se procedió a realizar un análisis de sensibilidad que permita verificar cómo se comporta frente a los cambios. Para este análisis, en primer lugar, se modificó la localización de los paraderos ya seleccionados por el modelo de manera tal de observar cómo se comporta el flujo vehicular respecto de estos cambios. En segundo lugar, para el análisis de sensibilidad se modificó la demanda total de pasajeros en los paraderos. En este caso, se aumentó el doble la cantidad pasajeros que ocupan el transporte público para así observar cómo se comporta el sistema en general ante un aumento en la demanda de los peatones.

\subsection{Propuesta de localización y discusión}

En la Fase 1 se observa una mejora general de los indicadores, tanto de los vehículos como de los peatones con la nueva localización del paradero que funcionará durante los desvíos. Si bien, estas mejoras son leves y no superan el $7 \%$ con respecto a los resultados del modelo de localización, se debe destacar que el cambio beneficia los indicadores de los peatones que son una variable importante dentro del modelo de localización. Por lo tanto, para la Fase 1 se optó por dejar los cambios introducidos y localizar el paradero en la intersección de René Schneider con J.M. Irarrázaval.

En cuanto a la Fase 2 existen dos escenarios: los días en que no hay Feria y los días jueves y domingo en que funciona la Feria en Avenida 5 de Abril. Para el caso de los vehículos en ambos escenarios hubo mejora en los indicadores de la velocidad promedio como de los tiempos de viaje de la red en general. Si bien, las colas de vehículos promedio mejoraron en ambos casos, especialmente en algunas calles donde la congestión se ve afectada en parte por la localización de los paraderos, estas mejoras no son significativas.

Así, la localización propuesta para el Plan de Desvíos debido al proyecto vial de mejoramiento del eje vial de Avenida 5 de Abril - Esquina Blanca con Avenida Del 
Ferrocarril se muestra a continuación en la Figura 7 ;Error! No se encuentra el origen de la referencia..

Fig. 7 - Propuesta de Localización de Nuevos Paraderos

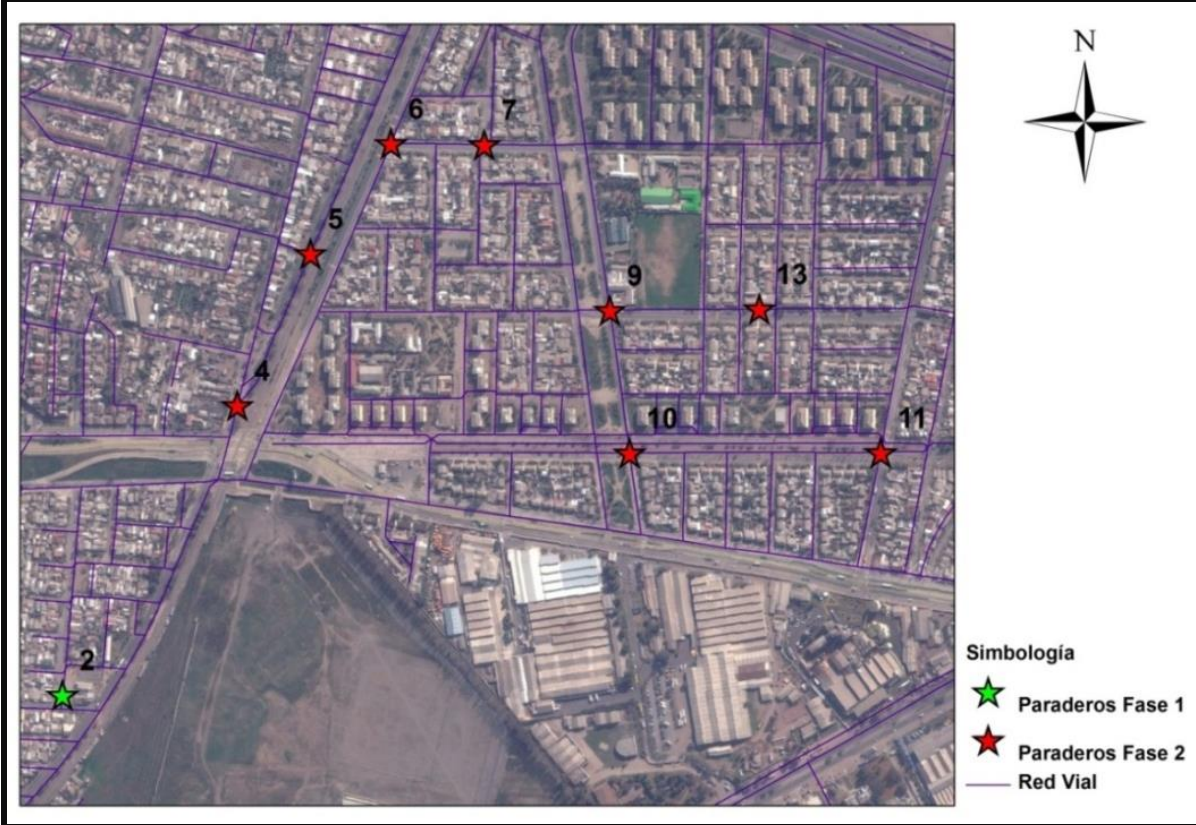

\section{CONCLUSIONES}

El presente trabajo presenta una metodología de localización de paradas de transporte público para proyectos viales que implica la implementación de un Plan de Desvíos producto de las obras que pretende realizar dicho proyecto. Para esto, la metodología se sirve de un modelo de optimización matemática que localiza de manera óptima los paraderos de transporte público en las vías alternativas propuestas por el Plan de Desvíos.

La principal variable de este modelo son los pasajeros que utilizan el transporte público, en base al tiempo que estos demoran en recorrer la distancia desde sus lugares de orígenes a las paradas, dicho modelo localiza de manera óptima las nuevas paradas que serán ubicadas en calles por donde pasarán los desvíos.

Esta metodología además, es aplicada en un caso real para comprobar su validez y determinar cómo se comporta en casos reales. Esto se logra a través de la simulación de diversos escenarios a partir de los resultados obtenidos por el modelo de localización.

La simulación entrega datos de los tiempos de viaje de los peatones desde su hogar hacia las paradas propuestas, y viceversa, basados en la velocidad de caminata de una persona estándar. Se determinó que las distancias que recorren las personas hasta los paraderos apenas supera los 300 metros e incluso, en el caso de la situación con proyecto, una vez iniciadas las obras viales y los desvíos, los resultados son levemente mejores que el caso con ubicaciones originales de las paradas. 
Solo los resultados de la simulación, en los días que hay feria, son levemente diferentes del resto de los datos, pero esto tiene que ver con la naturaleza de los desvíos, pues en estos días el flujo vehicular, incluido el transporte público, debe circular por calles que se alejan de los centroides de población del área de estudio, lo que obliga a los peatones a desplazarse lejos de sus lugares de residencia.

Aun así, el modelo aporta a minimizar dichas distancias y los resultados se mantienen dentro de lo esperado, pues las personas no superan los 400 metros caminando, lo que es una distancia aceptable para accesibilidad al TP.

Finalmente, esta propuesta metodológica es un aporte al área de la planificación de proyectos de vialidad, para la toma de decisiones por parte de los organismos pertinentes y de los encargados de la elaboración de dichos proyectos, así como de las partes evaluadoras. Si bien existen manuales y reglamentos en Chile, como el Manual de Diseño y Evaluación Social de Proyectos de Vialidad Urbana del Ministerio de Transporte, el cual es una guía detallada de cómo se deben elaborar los proyectos de vialidad en el país, éste no trata específicamente como deben localizarse los paraderos de transporte público cuando hay propuesto un Plan de Desvíos producto de las obras que se desean realizar. Con el presente trabajo, se demuestra que la localización de paraderos debe ser incorporada como un elemento importante en la normativa. Por lo tanto, esta metodología se vuelve un aporte a la toma de decisiones, para afectar lo menos posible a las personas, y las condiciones del servicio de transporte público y así evitar las incomodidades que traen este tipo de obras.

\section{AGRADECIMIENTOS}

Esta investigación fue apoyada y financiada a través del Proyecto $\mathrm{N}^{\circ}$ 061312MT del Departamento de Investigaciones Científicas y Tecnológicas (DICYT) de la Vicerrectoría de Investigación, Desarrollo e Innovación de la Universidad de Santiago de Chile (USACH).

\section{REFERENCIAS}

CHIEN, S., QIN, Z., LIU, R. (2004). Optimization of bus stop locations for improving transit accessibility. Transportation Planning and Technology 27(3), 211-227.

CORTÉS, C., BURGOS, V., FERNÁNDEZ, R. (2010). Modelling passengers, buses and stops in traffic microsimulation: review and extensions. Journal of Advanced Transportation 44(2), 72-88.

DOWLING, R., SKABARDONIS, A. Y ALEXIADIS, V. (2004). Traffic Analysis Toolbox Volume III: Guidelines for Applying Traffic Microsimulation Software. Federal Highway Administration, Report FHWA-HRT-04-040. 
GIPPS, P. (1981). A behavioural car-following model for computer simulation. Transportation Research 15(2), 105-111.

HIDAS, P., AITKEN, S., SHARMA, S., XU, M. (2009). Evaluation of Bus Operations by Microsimulation in a Sidney CBD Corridor. Australasian Transport Research Forum. Auckland.

IOANNOU A. y BOSE, P. (2000). Environmental evaluation of intelligent cruise control (ICC) vehicles. Intelligent Transportation Systems, Proceedings. 2000 IEEE, 352-357.

MAY, A. (1990). Traffic flow fundamentals. Prentice-Hall, New Jersey.

MEDINA, M. (2011). Localización Óptima de Paradas de Buses en Corredor de Transporte Público en Base a Estructura de Viajes en Múltiples Períodos. Aplicación en Corredor de Transporte Público de Avenida Grecia. Proyecto para optar al grado de Magíster en Ingeniería. Pontificia Universidad Católica de Chile.

MEDINA, M., GIESEN, R., MUÑOZ, J.C. (2013). Model for the Optimal Location of Bus Stops and Its Application to a Public Transport Corridor in Santiago, Chile. Transportation Research Record: Journal of the Transportation Research Board 2352(3), 84-93.

MOURA, J. L., ALONSO, B., IBEAS, A., RUISÁNCHEZ, F. J. (2012). A Two-Stage Urban Bus Stop Location Model. Networks and Spatial Economics 12(3), 403-420.

PAPAGEORGIOU, G., DAMIANOU, P., PITSILlIDES, A., APHAMIS, T., IOANNOU, P. (2009). Modelling and Simulation of Transportation Systems: Planning for a Bus Priority System. Networks Research Laboratory, University of Cyprus.

RONALDO, A. y ISMAIL, M. (2012). Comparison of the two Microsimulation Software AIMSUN \& SUMO for Highway Traffic Modelling. Linkoping University.

SYKES, P. (2007). Transport Planning with Microsimulation. Journal of Maps 3(1), 122134.

OLSTAM J. Y TAPANI, A. (2004). Comparison of Car-Following Models. Publication of Swedish National Road and Transport Research Institute. 